\title{
Radiofrequency Catheter Ablation of Left-sided Accessory Pathways Using a Transeptal Technique and Specialized Long Intravascular Sheaths
}

\author{
Efficacy, Recurrence Rate and Complications
}

\author{
Alex S.B. YIP, MBBS, FRCP(Ed), \\ Wing-Hing CHow, MBBS, FRCP(Ed), \\ Tak-Cheung YUNG, ${ }^{1}$ MBBS, MRCP (UK), \\ Elaine M.C. CHAU, MBBS, MRCP(UK), \\ and Tsan-Fai Chan, MBBS, MRCP(UK)
}

\begin{abstract}
SUMmary
49 patients with 51 left-sided accessory pathways underwent radiofrequency catheter ablation for symptomatic supraventricular tachycardia via the transeptal route using specialised long vascular sheaths with compound curves. The procedure was successful in 45 patients $(92 \%)$. The mean fluroscopic time was $22.5 \pm 15.2 \mathrm{mins}$ and the mean procedure time was $1.7 \pm 0.5$ hours. Pericardial tamponade occurred in 2 patients $(4 \%)$ and 2 patients $(4 \%)$ required switching to the retrograde transaortic route for successful ablation of the pathways. During the period of follow-up of $16.8 \pm 6.9$ months, clinical recurrence occurred in 2 patients $(4 \%)$. In conclusion, the transeptal route of radiofrequency catheter ablation is a useful alternative strategy to the transaortic approach with good long term results. The use of specialised sheaths may help in stabilisation of the catheter during the procedure which can generate more adequate lesions and consequently a lower recurrence rate. (Jpn Heart J 1997; 38: 643-650)
\end{abstract}

Key words: Supraventricular tachycardia, Accessory pathways

$\mathrm{R}$

ADIOFREQUENCY (RF) ablation has emerged as the curative treatment for patients with reentrant arrhythmias utilising accessory pathways and a success rate of over $95 \%$ has been reported, especially at specialised centres." Transaortic approach was initially the method used to ablate left-sided accessory pathways but advocates of the transeptal method argue that the latter should be

From the Department of Cardiology, and 'Department of Paediatric Cardiology, the Grantham Hospital, Aberdeen, Hong Kong.

Address for correspondence: Alex S. B. Yip, MD, Department of Cardiology, the Grantham Hospital, Aberdeen, Hong Kong.

Received for publication Feburary 17, 1997.

Accepted April 28, 1997. 
equally useful, if not superior to the former. ${ }^{2)}$ Initial experience with the transeptal approach was performed in patients using standard transeptal sheaths ${ }^{2,3)}$ with encouraging results. Subsequently the development of specialised long vascular sheaths with compound curves aims to stabilise the catheter and to facilitate mapping and ablation along the mitral annulus during the procedure, and hence may reduce the procedure time and the subsequent recurrence rate. We report our experience with the transeptal approach using these specialised sheaths in the RF ablation of left-sided pathways targeting the atrial insertion sites, including its complications and recurrence rate on follow-up.

\section{Patients and Methods}

Between May 1994 and February 1996, 49 consecutive adult patients with left-sided accessory pathways underwent a radiofrequency ablation procedure via the transeptal route as the initial approach in the cardiac catheterisation laboratory. All patients had recurrent documented symptomatic tachycardia not controlled with pharmacological agents. The investigation protocol was approved by the Research and Ethics Committee of the Grantham Hospital. All patients gave their informed consent prior to the procedure and were sedated with intravenous midazolam during the procedure. Quadripolar catheters were placed in the high right atrium, His bundle region and right ventricular apex and a $5 \mathrm{~mm}$ interspace decapolar catheter (Bard Inc., Billerica, MA, USA) was inserted into the coronary sinus for detailed mapping of the mitral annulus during tachycardia or ventricular pacing.

Each patient underwent a standard diagnostic electrophysiologic study prior to the RF catheter ablation procedure. A baseline electrophysiologic study was performed using methods described previously. ${ }^{4)}$ Accessory pathway localization was achieved by analysis of the earliest retrograde atrial activation during orthodromic atrioventricular tachycardia induced or with ventricular pacing in the intracardiac electrograms. Accurate epicardial identification of the atrial insertion site was performed with coronary sinus mapping during tachycardia, taking the shortest ventriculo-atrial interval recorded from the pair of bipolar electrograms of the coronary sinus catheter as the site of atrial insertion of the accessory pathway. Endocardial mapping and localization of the accessory pathway atrial insertion site was accomplished with the transeptal approach using a modified Brockenbrough needle. ${ }^{2)}$ After transeptal puncture, heparin was given using a 3,000 unit bolus and 1,000 unit per hour during the procedure. After localisation of the atrial insertion site with coronary sinus mapping, the appropriate type of specialised long vascular sheath with different curvatures (Fast-Cath left heart series introducers, Daig Gorp, Minnetonka, MN, USA) was then cho- 


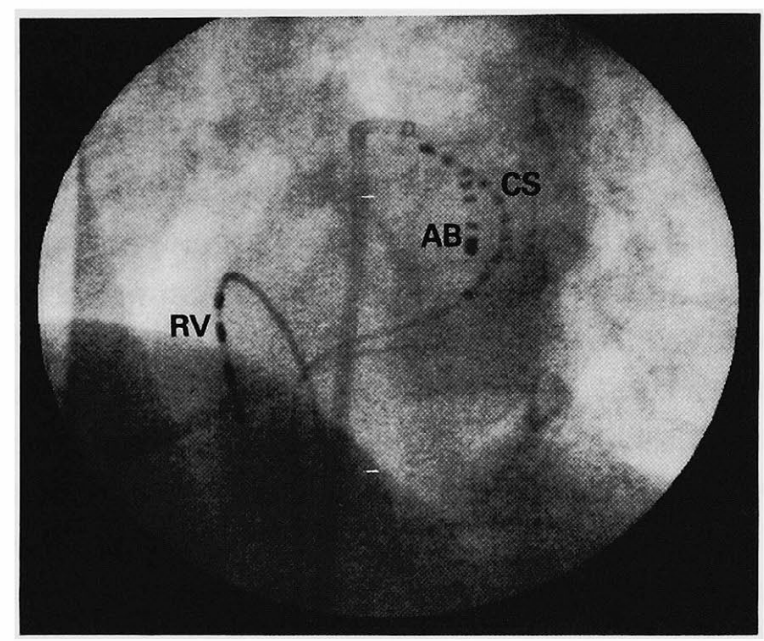

Figure 1. Left anterior oblique (40 degrees) fluoroscopic view showing the mapping/ablation catheter with the long intravascular sheath in situ at the successful site of ablation of the atrial insertion site of a left lateral concealed accessory pathway. Decapolar catheter in coronary sinus and quadripolar catheter in right ventricle are also shown.

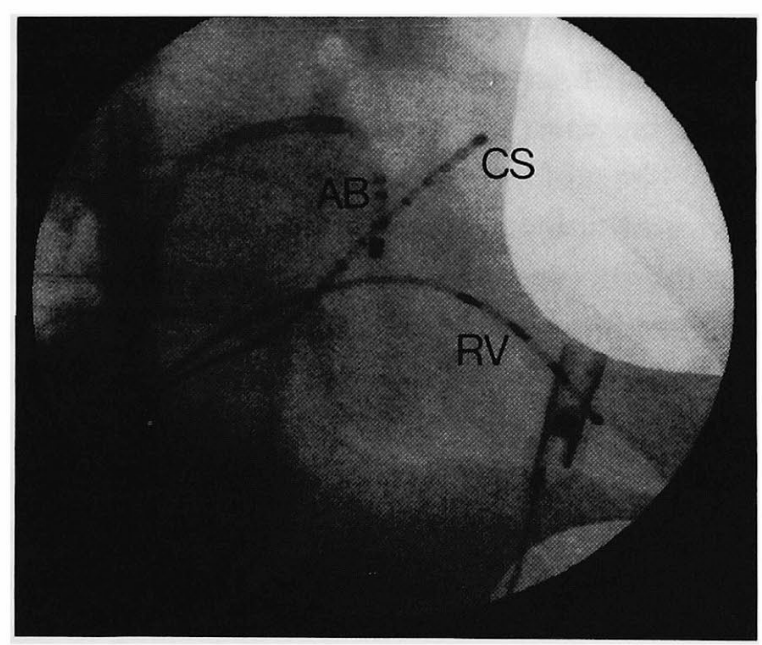

Figure 2. Right anterior oblique (30 degrees) fluoroscopic view showing the same catheter positions as in Figure 1.

sen according to the following guidelines: SLl for anterolateral, SL2 for lateral, SL3 for posterolateral and SL4 for posteroseptal pathways. Through the sheath the mapping/ablation catheter was passed into the left atrium (Figure 1). Endocardial mapping was performed using a steerable 7F $4 \mathrm{~mm}$ tip electrode catheter with 2-5-2 mm interelectrode spacing (Medtronic CardioRhythm, CA, USA). 
Bipolar (30-500 Hz bandpass filter) and distal electrode unipolar $(0.01-500 \mathrm{~Hz}$ bandpass filter) electrograms were continuously recorded from the ablation catheter. Endocardial accessory pathway atrial insertion site was identified using atrial electrogram polarity reversal ${ }^{5)}$ which has been shown to be highly effective in this approach of ablation. Accessory pathway potentials were not routinely looked for. Catheter stability was assessed from the consistency of local atrial and ventricular electrogram amplitudes and ratios. ${ }^{2)}$

RF ablation was performed using unmodulated $500 \mathrm{KHz}$ alternating current generated from a standard unit (Atakr Ablation System, Medtronic, MN). Energy was delivered in a monopolar fashion between the ablating catheter tip electrode and a skin electrode. Temperature control mode was used in all patients and the power output was automatically varied to achieve target electrode temperatures $\left(<70^{\circ} \mathrm{C}\right.$ ) using closed-loop control. Radiofrequency energy applications were delivered for 90 seconds unless the catheter position had changed during the application, a sudden rise of impedance occurred or there was no effect after 10 seconds of application. The ablation catheter position was monitored continuously using fluoroscopy throughout each energy application to ensure stable catheter position and the appropriate delivery of energy to the targeted tissue. Repeat mapping and ablation attempts were necessary in some patients until accessory pathway block was achieved. After successful ablation the patient was observed in the laboratory for 20 minutes when a full anterograde and retrograde study for the evaluation of the accessory pathway conduction capacity was made. The ablation procedure was considered successful when there was no evidence of accessory pathway conduction for at least 20 minutes after the delivery of the successful pulse of RF energy.

Ablation procedure time was defined as the start of local anaesthetic injection for venous access to the end of post ablation electrophysiologic evaluation. All patients were monitored continuously on telemetry for 24 hours after the ablation procedure. Serial 12-lead ECGs was performed every 12 hours after the procedure for 36 hours and routine out-patient follow-up was made at 2 weeks, $1,2,3,6$ and 12 months and then yearly intervals. Aspirin (150 mg daily) was prescribed for all patients who were monitored according to their symptoms and using an ECG event recorder monitoring for arrhythmia documentation. Clinical recurrence is defined as documented supraventricular tachycardia or a return of pre-excitation on 12-lead ECG during follow-up. Results are presented as mean \pm standard deviation.

\section{Results}

A total of 51 pathways in 49 patients (30 males and 19 females, mean age 
$33.2 \pm 13.5$ years; range $16-64$ years) with left-sided accessory pathways underwent attempted ablation. Twelve patients have manifest pre-excitation, i.e. Wolff-Parkinson-White syndrome and the remaining were concealed pathways. Two patients have multiple concealed left-sided pathways (both have a combination of left lateral and left posteroseptal pathways). The distribution of the accessory pathway locations was as follows: posteroseptal in $6(12 \%)$, posterior in 5 $(10 \%)$, lateral in $36(71 \%)$, anterolateral in $3(6 \%)$ and a broad band of accessory pathway fibres in $1(2 \%)$. The procedure was successful in 45 patients $(92 \%)$. The procedure time was $1.7 \pm 0.5$ hours with a mean fluoroscopic time of $22.5 \pm 15.2$ minutes. The mean number of RF pulses delivered was $4.1 \pm 3.6$ (range $1-20$ ). The patients were followed-up for $16.8 \pm 6.9$ months.

Complications and failures: For the 4 patients whose pathways were unsuccessfully ablated using the transeptal route, 2 patients (4\%) developed pericardial tamponade during the procedure necessitating immediate resuscitation and termination of the procedure. In both patients the procedure was attempted during our early experience with the procedure (cases 5 and 8 ) after prolonged manipulation of the catheter in the left atrium ( $>3$ hours). Both patients were successfully treated with percutaneous drainage. Repeat ablation attempted via the retrograde transaortic route within 2 months successfully eliminated the accessory pathway conduction in both patients. No other complication including heart block, myocardial infarction or systemic embolism occurred in any patient.

Two patients required switching to the retrograde transaortic approach for successful ablation of their pathways after prolonged unsuccessful effort via the transeptal route ( $>3$ hours). One had severe emphysema and a vertically displaced mitral annulus. However, there was no apparent reason for the transeptal failure in the other patient who had a single left lateral concealed pathway with otherwise normal cardiac anatomy.

Documented clinical recurrence occurred in 2 patients $(4 \%)$. Both patients had documented attacks of supraventricular tachycardias within 3 months after the initial successful ablation. The location of the accessory pathways were left lateral (concealed) in 1 and a broad band of concealed accessory pathway fibres in the other. Repeat ablation procedure via the transeptal route was successful in both patients.

\section{Discussion}

Retrograde transaortic approach was the "standard" approach for the ablation of left-sided accessory pathways using RF energy. However, there were concerns regarding possible trauma to the aortic valve during the procedure, ${ }^{6)}$ the creation of lesions within the ventricle, and increased complications resulting 
from prolonged manipulation within the arterial system. Furthermore, manipulation of the mapping/ablation catheter within the trabeculated ventricle can be difficult at times when fine control of movements are necessary. Since Swartz ${ }^{2)}$ published his initial report on the transeptal approach to ablate the atrial insertion sites, this approach has been adopted by many cardiac electrophysiologic centres to ablate left-sided accessory pathways, especially the concealed pathways. Some centres consider it the method of choice since it can be more speedy, can facilitate finer control of the mapping/ablation catheter along the smooth atrial wall, and avoids the creation of lesions in the ventricle which may potentially generate ventricular arrhythmias in the future. Some electrophysiologists consider it to be particularly suitable in small patients and in the young. ${ }^{3)}$ The success and recurrence rate reported in their series were $94 \%$ and $8.7 \%$ respectively, ${ }^{2)}$ which were comparable to the results from the retrograde transaortic approach. However, in the absence of these specialised sheaths the procedure time and fluoroscopic time in their series were $4.9 \pm 2.2$ hours and $62.2 \pm 47.1$ minutes, respectively, which are significantly longer when compared with our series.

The development of long vascular sheaths with compound curves was designed to follow the contour of the atrial aspect of the left atrium above the mitral annulus. The aim is to facilitate mapping of atrial signals, to avoid excessive manipulation during the procedure, to improve catheter stability and to improve torque transmission from the catheter handle to the tip of the catheter during ablation. This serves to limit unnecessary manipulation of the catheter within the thin-walled left atrium, which may otherwise produce cardiac perforation resulting in pericardial tamponade. Through these specialised sheaths the ablation catheter can be brought into close proximity of the atrial insertion site of the accessory pathway thus facilitating speedy mapping and ablation attempts. This is the first reported series on this approach of transeptal ablation using these specialised sheaths with compound curves.

Recurrence rate after successful ablation of left-sided accessory pathways using the retrograde transaortic approach varies between $3.3-8 \%$. . $^{\text {) }}$ Although the number of patients in our series is relatively small, our clinical recurrence rate of $4 \%$ is lower when compared with 2 published series ${ }^{2,3)}$ using the transeptal approach ( $8.7 \%$ and $9 \%$ respectively) and also compares favourably with other series using the retrograde transaortic approach. This may reflect the beneficial "stabilising" effect by the specialised sheath, thus enabling better tissue contact and generation of a more adequate lesion by the RF energy. Routine electrophysiologic study was not performed in patients who are asymptomatic after successful RF ablaion. As recurrence of accessory pathway conduction is very unusual after 6 months post successful ablation ${ }^{7)}$ and all the patients is our 
series have been followed up for at least 6 months, the clinical recurrence rate is a good indication of the true curative rate of the procedure. Further studies are necessary to confirm the findings. In addition, the procedure time in our patients compares favourably with the retrograde transaortic series ${ }^{8)}(1.7 \pm 0.5$ hours vs. $2.2 \pm 1.3$ hours, respectively). Our short fluoroscopic time of $22.5 \pm 15.2 \mathrm{~min}$ has the advantage of reducing radiation exposure for patients and medical/nursing personnel.

Our experience with the patient with an emphysematous chest and a vertically displaced heart suggested that in this group of patients the transeptal route for RF ablation may be less suitable. The ablation catheter has to be curved vertically downwards through an awkward angle in order to reach the displaced mitral annulus. In that patient even removal of the sheath did not result in bringing the catheter close to the mitral annulus and a different approach, namely the retrograde transaortic approach, was required to successfully ablate the pathway.

In both patients who developed pericardial tamponade the procedure time was relatively prolonged ( $>3$ hours) which involved increased manipulation of the catheter, causing trauma to the atrial wall. Hence, prompt consideration should be given to switch to the retrograde transaortic route if initial effort via the transeptal route could not abolish accessory pathway conduction within a reasonable time frame (e.g. 2 hours). In addition, the transeptal route is particularly useful in patients in whom ablation via the transaortic route is not possible or potentially hazardous, e.g. patients with aortic stenosis, aortic valve replacement or severe peripheral arterial disease.

In conclusion, ablation of the accessory pathway via the transeptal route is an effective method and should be considered complementary to the retrograde transaortic approach when performed by a cardiologist who is experienced in the transeptal puncture technique. The use of specialised intravascular sheath may provide an additional stabilisation effect during mapping and ablation, resulting in shorter procedurc and fluoroscopic times and lower recurrence rate. Careful patient sclection and avoidance of prolonged ineffective ablation can help minimize complications.

\section{REFERENGES}

1. Jackman WM, Wang X, Friday KJ, et al. Catheter ablation of accessory pathways (Wolff-ParkinsonWhite syndrome) by radiofrequency current. N Engl J Med 1991; 324: 1605-11.

2. Swartz JF, Tracey CM, Fletcher $\mathrm{RD}$. Radiofrequency endocardial catheter ablation of accessory atriovenetricular pathway atrial insertion site. Circulation 1993; 87: 487-9.

3. Saul JP, Hulse E, W De, et al. Catheter ablation of accessory atrioventricular pathways in young patients: use of long vascular sheaths, the transeptal approach and a retrograde left posterior parallel approach. J Am Coll Gardiol 1993; 21: 571-83. 
4. Ross DL, Denniss AR, Uther JB. Electrophysiologic study in supraventricular arrhythmias. In: Schroeder JS, Brest AV, editors Invasive Cardiology. Cardiovascular Clinics. Philadelphia: FA Davis, 1985: 187-213.

5. Swartz JF, Fisher WG, Tracey CM. Ablation of left sided atrioventricular accessory pathways via the transeptal atrial approach. In: Huang SKS, editor. Radiofrequency catheter ablation of cardiac arrhythmia. New York: Futura 1995: 251-75.

6. Seifort MJ, Morady F, Calkins HG, Langberg JJ. Aortic leaflet perforation during radiofrequency ablation. Pacing Clin Electrophysiol 1991; 14: 1582-5.

7. Langberg $\mathrm{JJ}$, Calkins $\mathrm{H}, \mathrm{Kim} \mathrm{YN}$, et al. Recurrence of conduction in accessory atrioventricular connections after initially successful radiofrequency catheter ablation. J Am Coll Cardiol 1992; 19: 158892.

8. Calkins H, Langberg J, Sousa J, et al. Radiofrequency catheter ablation of accessory atrioventricular connections in 250 patients; abbreviated therapeutic approach to Wolf-Parkinson-White syndrome. Circulation 1992; 85: 1337-46. 